\title{
On the interpretations of joint modelling in community ecology
}

\author{
Giovanni Poggiato ${ }^{1,2,}{ }^{*}$, Tamara Münkemüller ${ }^{1}$, Daria Bystrova ${ }^{1,2}$, Julyan Arbel ${ }^{2}$, \\ James Clark ${ }^{3,4,5}$ and Wilfried Thuiller ${ }^{1}$ \\ ${ }^{1}$ Univ. of Grenoble Alpes, CNRS, Univ. Savoie Mont Blanc, LECA, Grenoble, France \\ ${ }^{2}$ Univ. of Grenoble Alpes, Inria, CNRS, Grenoble INP, LK, Grenoble, France \\ ${ }^{3}$ Univ. of Grenoble Alpes, Irstea, LESSEM, Grenoble, France \\ ${ }^{4}$ Nicholas School of the Environment, Duke University, Durham, North Carolina \\ 27708 USA \\ ${ }^{5}$ Department of Statistical Science, Duke University, Durham, North Carolina \\ 27708 USA \\ *Corresponding author: giov.poggiato@gmail.com
}

Explaining and modelling species communities is more than ever a central goal of ecology. Recently, joint species distribution models (JSDMs), which extend species distribution models (SDMs) by considering correlations among species, have been proposed to improve species community analyses and rare species predictions while potentially inferring species interactions. Here, we illustrate the mathematical links between SDMs and JSDMs and their ecological implications and demonstrate that JSDMs, just like SDMs, cannot separate environmental effects from biotic interactions. We provide a guide to the conditions under which JSDMs are (or are not) preferable to SDMs for species community modelling. More generally, we call for a better uptake and clarification of novel statistical developments in the field of biodiversity modelling.

\section{Keywords}

Biodiversity modelling, Biotic interactions, Community data, Environmental niche, Joint Species Distribution Models 
In an era of global changes, developing reliable biodiversity models has become an important research area.

Species distribution models are the common tools to understand and predict the distributions of species across space and time. However, they fail to explicitly account for species interactions.

To this aim, joint species distribution models were introduced to tease apart the effect of the environment from that of species interactions, to improve rare species modelling, to account for functional traits, and to improve the predictive power of biodiversity models.

Nevertheless, most announced advantages have remained unfulfilled, and there is still a need to better integrate the effect of species interactions in the response of species to environmental change.

\section{Glossary}

Covariates: variables used to predict the response variables (see below). In this paper covariates represent the abiotic conditions. A missing covariate is a variable that is not included in the model but has an important effect on the response variables.

Generalized linear model (GLM): a flexible generalization of ordinary linear regression to predict a response variable from a distribution in the exponential family (Poisson, binomial, etc.), and assuming that some known transformation of the mean response is a linear function of predictor variables.

Hierarchical model: a statistical model written in multiple levels (hierarchical form). Hierarchical modeling allows sharing information between entities (mostly species here) to facilitate parameter estimation, an advantage commonly referred to as 'borrowing strength'.

Latent variable: a variable not directly observed and usually introduced to model correlations between response variables.

Niche (fundamental, sensu Hutchinson): the physiological dependence of the species on the environment.

Niche (realized, sensu Hutchinson): the observed relationships between the species and the environment. This is the outcome of both the environmental effect and biotic interactions.

Conditional predictions: the prediction of the distribution of the value(s) of one or more response variable(s), given the value(s) of one or more other response variable(s). Conditional predictions could be derived through the use of the residual correlation matrix (see below).

Joint predictions: the prediction of the distribution of the joint values of two or more response variable(s). Joint predictions could be derived through the use of the residual correlation matrix (see below).

Marginal predictions: the prediction of the distribution of the value(s) of one or more response variable(s), irrespective of the value(s) of one or more other response variable(s). Marginal predictions are the typical output of SDMs and JSDMs.

Regression coefficients: the parameters that describe the relationships between the response variables and the covariates. In (joint) species distribution models, they are interpreted as descriptions of species' niches.

Residual correlation matrix: the correlation matrix between the response variables (see below) after accounting for the effect of the covariates.

Response variables: the variables of interest to be modelled and predicted. In this article, they mostly represent the presence-absences of species. 


\section{From ecological theory to biodiversity modelling}

73

Understanding the ecological processes driving the distribution of life on Earth has always been a central goal in ecology. This is more than ever crucial to project how biodiversity from various ecosystems will respond to global changes. Researchers have long focused on the description of how species are spatially distributed and on the main drivers explaining these distributions (Van Humboldt, early 1800s). It is now clear that three fundamental ecological processes determine whether a species can occupy a site and maintain viable populations: limitation by abiotic conditions, biotic interactions and dispersal limitation (see Box 1, [1-3]).

While we theoretically know the complex processes that shape communities, their relative importance is generally unknown, making it difficult to predict how these communities will respond to environmental changes [4]. Statistical ecology has arisen as a discipline that moves away from describing biodiversity patterns towards modelling the output of the ecological processes that generate these patterns [5]. Notably, the so called biodiversity models predict the distribution and abundance of multiple species based on a set of environmental conditions [6]. To properly interpret the parameters of these models, and to guarantee the quality and reliability of their predictions, it is key to understand how they integrate the fundamental ecological processes shaping species ranges and community structure [6].

Species distribution models (SDMs, [7]), the most common statistical tool to model species distributions, early on raised debates on how to interpret their parameters in light of ecological processes. SDMs relate the presence-absence or the abundance of a species to environmental covariates (see Glossary) and use this relationship to predict its distribution in space and/or time [8]. Originally, most SDMs relied on generalized linear models (GLMs, [9]), with the deterministic regression coefficients for the relationship of the species with the environment, and a residual part for the unexplained variation. Usability and increasing data availability have boosted the use of SDMs [10] in ecology and conservation. However, by modelling the observed species-environment relationship for each species independently, they only capture the combined effects of both abiotic and biotic environments (i.e. the so-called realized niche, see Glossary). The pure effects of the abiotic environment are not separated from the effects of species interactions and the fundamental niche remains unknown [11], which potentially distorts predictions [12]. Despite these issues, SDMs were also used to predict communities by summing over single species predictions (e.g. stacked SDMs, $[13,14]$ ), eventually with some additional constraints to account for biotic filters $[15,16]$. However, this two-step procedure allows neither for error propagation nor for joint parameter estimations and is conceptually flawed as the realized niche estimated from SDMs inherently accounts for biotic constraints.

In the last decade, multi-species distribution models (MSDMs) and joint species distribution models (JSDMs) were introduced to overcome the assumption of SDMs that species' distributions are independent of each other. MSDMs are extensions of GLMs, where the estimated species-environment relationship between species are connected [17]. By modelling the regression coefficients hierarchically, they consider commonalities between species, so that, for instance, species with similar traits respond similarly to the environment [18-21]. As a result, rare species could 'borrow strength' from common species if they do not behave fundamentally differently [17]. JSDMs, as a further extension of GLMs (but 
see $[22,23]$ for other approaches), infer a correlation matrix from the residuals (hereafter residual correlation matrix)

108 that reflects species co-occurrence patterns not explained by the environmental predictors [24]. Residual correlations 109 may arise from model mis-specifications, missing covariates or species interactions (Box 2, review in [25-27]). Thus, 110 JSDMs intuitively have been proposed to simultaneously explore, and potentially disentangle, limitations by abiotic 111 conditions and biotic interactions [25]. Although these new statistical models are receiving increasing attention, there is 112 so far a lack of clarification on both the ecological processes they incorporate and on their specific commonalities and 113 advantages with respect to SDMs. Some of the widespread beliefs, such as the idea that JSDMs can "account for biotic 114 interactions in species distribution models" [28], have never been proven.

115 In this paper, we first reunify SDMs, MSDMs and JSDMs under a common notation to better identify their similarities and 116 differences (Box 2). Like MSDMs, JSDMs can also model the regression coefficients hierarchically, but since this is not 117 always implemented (see [28,29]), we consider here JSDMs and MSDMs as two different extensions of SDMs. Second, 118 we tease apart the true advantages of JSDMs from false beliefs and possible misinterpretations, therefore allowing to 119 interpret these models in the light of fundamental ecological processes. Specifically, we address the following questions:

120 1. Can JSDMs and MSDMs improve the estimation of species' fundamental niches?

121 2. What can the residual correlation matrix tell us about biotic interactions?

122 3. When and why do JSDMs outperform SDMs?

123 This opinion piece differs from previous papers on JSDMs in that we neither introduce new methodological developments, 124 nor compare these models with data. Instead, we rigorously and mathematically demonstrate how to interpret MSDMs 125 and JSDMs, providing a guide on why and when these models should be preferred to SDMs. Our aim is to enable users to 126 serenely choose and apply these models to make the best of their potential. 

3]):

- the species has to physically reach a site, i.e. to access a region [53];

- the abiotic environmental conditions (e.g. temperature or soil pH) must be physiologically suitable for the species;

- the biotic environment (i.e. interactions with other species) must be suitable for the species.

The first condition is a matter of species' capacity to disperse to a site from other occupied areas. It is related to the biogeographic history of the species, and thus to all factors limiting its distribution from the place where it first originated, such as barriers to migration, biotic and abiotic dispersal vectors or rare long-distance dispersal events. The second condition depends on abiotic conditions, which means that the combination of abiotic environmental variables at the site are within the range of environmental conditions that the species requires to grow and maintain viable populations. These suitable environmental conditions represent the species' fundamental niche [54]. The third condition concerns biotic interactions, i.e. interactions with other organisms, either neutral, positive or negative, symmetric or asymmetric, which themselves are influenced by the environment through their influence on all organisms in the local community. The environmental conditions where a species can therefore survive accounting for other species are called the species' realized niche [54]. This is what we observe when sampling the distribution of a species in the wild. In a given site, these processes influence all species from the regional pool to create local communities that represent a relevant scale to investigate biodiversity distribution (e.g. few square meters for plants, a soil core for microbes) $[6,55]$.

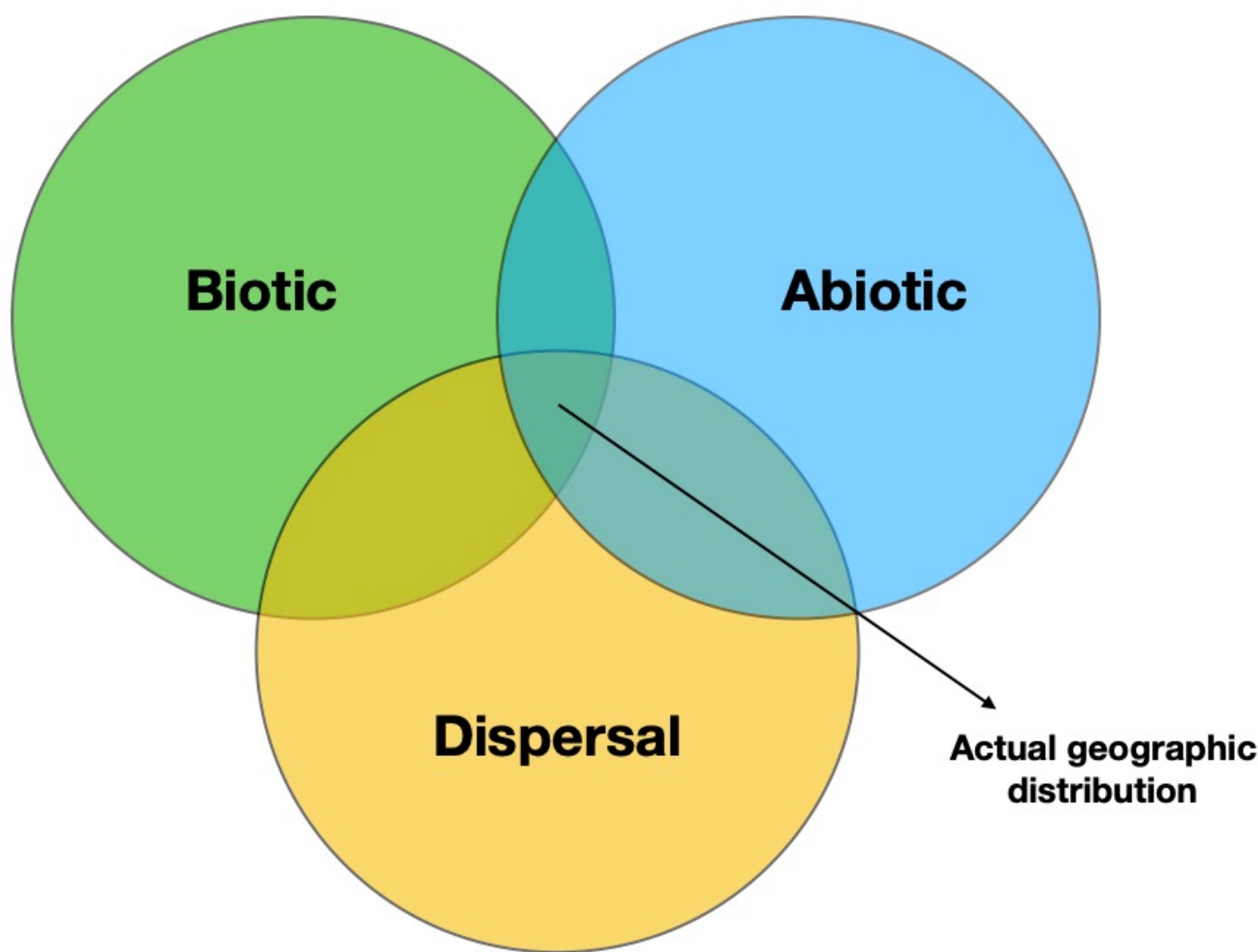

Figure I. The three factors that shape the observed species distribution [3]. The blue circle describes the fundamental niche, while the realized niche is represented by the intersection of the green and blue circle. 


\section{Box 2: Mathematical notations from SDMs to JSDMs}

Focusing on presence-absence data, the response variable $y_{i j}=1$ if species $j=1, \ldots, S$ is present at site $i=1, \ldots, n$ and 0 otherwise. All models relate the S-dimensional vector $\boldsymbol{y}_{i}$ to a set of $K$ environmental covariates $\boldsymbol{x}_{i}=\left\{x_{i k}\right\}_{k=1}^{K}$.

\section{SDMs}

GLMs can model presence-absence data using a probit link. Probit regression can be described as a latent variable model with the probability of presence being modelled as the probability of a latent Gaussian variable to be positive [56]. Each species $j$ is modelled independently, with:

$$
\begin{aligned}
y_{i j} & =I\left(z_{i j}>0\right) \\
z_{i j} & =\boldsymbol{\beta}_{j}^{T} \boldsymbol{x}_{i}+\varepsilon_{i j} \\
\varepsilon_{i j} & \stackrel{i i d}{\sim} N(0,1)
\end{aligned}
$$

where $I()$ is the indicator function and $N(0,1)$ is the standard univariate Gaussian distribution. The variance term is restricted to 1 for identifiability reasons. The regression coefficients $\boldsymbol{\beta}_{j} \in \mathbb{R}^{K}$ give the response of species $j$ to the abiotic covariates [26]. The probability of species $j$ to be present at site $i$ is thus $\operatorname{probit}\left(y_{i j}=1\right)=\boldsymbol{\beta}_{j}^{T} \boldsymbol{x}_{i}$.

\section{MSDMs}

MSDMs model the regression coefficients of [I] hierarchically: $\boldsymbol{\beta}_{j} \stackrel{i i d}{\sim} N_{K}(\boldsymbol{\mu}, \boldsymbol{V})$, where $N_{K}$ is the multivariate Kdimensional Gaussian distribution. As a consequence, species' responses to the environment are shared across species, which can be of particular interest for rare species. Coefficients can also be constrained by trait and/or phylogenetic information (by including them in $\boldsymbol{\mu}$ and/or $\boldsymbol{V}$ ).

\section{JSDMs}

Most JSDMs extend GLMs in what is commonly called the multivariate probit model [57]. This model is based on the same latent variable idea as described above, but uses an S-dimensional vector:

$$
\begin{gathered}
y_{i j}=I\left(z_{i j}>0\right) \\
\boldsymbol{z}_{i}=\boldsymbol{\beta} \boldsymbol{x}_{i}+\boldsymbol{\varepsilon}_{i} \\
\boldsymbol{\varepsilon}_{i} \stackrel{i i d}{\sim} N_{S}(0, \boldsymbol{R})
\end{gathered}
$$

where $\boldsymbol{R}$ is a correlation matrix, and not a covariance matrix, for identifiability reasons. $\boldsymbol{R}$ describes the residual correlation among taxa, and reflects species co-occurrence patterns not explained by the selected abiotic covariates. $\boldsymbol{\beta}$ is a $K \times S$ matrix whose columns $\boldsymbol{\beta}_{j}$ are the species-specific response to the environment. Importantly, $\boldsymbol{R}$ does not affect the marginal probability of presence of each species, $\operatorname{probit}\left(y_{i j}=1\right)=\boldsymbol{\beta}_{j}^{T} \boldsymbol{x}_{i}$. Thus, marginal predictions only depend on the estimated regression coefficients for both SDMs and JSDMs [57].

Many JSDMs use latent factors to reduce the dimension of $\boldsymbol{R}$ (see Appendix A). JSDMS can also model the regression coefficients hierarchically, therefore integrating the advantages of MSDMs and obtaining highly flexible and complex models [e.g. 26].

\section{Reconciling SDMs, MSDMs and JSDMs}

Model [I] can be written in the same way as [II], but with a diagonal residual correlation $\boldsymbol{\varepsilon}_{i} \stackrel{i i d}{\sim} N(0, I)$. In other words, the only difference is that SDMs and MSDMs assume independent residuals, while JSDMs allow for correlations between them.

\section{Question 1: Can JSDMs and MSDMs improve the estimation of species'}

\section{fundamental niches?}

Characterizing the fundamental niches with observational data, teasing apart the effects of abiotic and biotic ecological processes on species distributions and community assembly, is a critical challenge to predict the future of biodiversity

$[6,12]$. Since they model multiple species together, we may believe that MSDMs and JSDMs can better fit the response of each species to environmental covariates by using information on the other species, and thus ultimately, may allow 
to retrieve the fundamental niche of species. JSDMs, in particular, have been repeatedly suggested to separate abiotic and biotic conditions and -if this suggestion was right- should allow to approach species' fundamental niches [25]. But can the models hold these promises? Below, we outline why this is not the case, neither for JSDMs nor for MDSMs.

In both SDMs and JSDMs, the species' niche (approximated by the regression coefficients) is estimated through minimizing species-specific regression residuals. In other words, should we infer a residual correlation matrix from the residuals (JSDMs) or not (SDMs), the estimated niches coincide. In Appendix B, we demonstrate that the estimates of the regression coefficients are identical for JSDMs and SDMs, at least for Gaussian data. The uncertainty around these estimates might differ, but it is difficult to prove whether one is always greater or lower than the other one. Extending this analytical proof to other data types is challenging. However, empirical comparisons for presence-absence data also showed no differences in the regression coefficients estimates between a comparable SDM and a JSDM approach (same package, same inference, only the estimation of correlation matrix differed, Box 3). Indeed, since JSDMs model the expected distribution of species as exclusively dependent on the environmental conditions (through the regression term), while all the other factors potentially influencing species' distributions (e.g., missing predictors, biotic interactions) can only impact the (co)variation (given by the residual correlations) around this expected value. In consequence, JSDMs, just like SDMs, do not control for the effect of other species when inferring species niches, and thus only retrieve the realized niches (see Appendix B for a further discussion). Importantly, it also means that for a set of modelled species, the correlations between the residuals of independent SDMs closely approximate the residual correlation matrix of a JSDM (Box 3), with the advantage of the latter to propagate model uncertainties in a more correct way and the former to be easier to apply ([30], page 11).

In contrast, MSDMs (and JSDMs with hierarchical coefficients) estimate different species niches than SDMs, especially for rare species. This is, however, not linked to species interactions. Thanks to the hierarchical part of the model, MSDMs share information between species [17], and can constrain, for example, two phylogenetically or functionally closely-related species to respond similarly to the environment (i.e. similar niches) [18]. Taking phylogeny and/or functional traits into account allows to test their importance in shaping species distribution [26]. MSDMs have been considered as a great improvement for modelling rare species for which niche estimates are difficult to obtain due to low sample size. Forcing niche estimates to resemble those of closely related common species circumvents this problem. However, this advantage only holds if rare and common species respond in the same way to the environment and leads to false estimates if this assumption is wrong. While the assumption may hold for hardly detectable species, there are many ecological reasons why truly rare species differ from common species in their response to the environment. Species can be rare because they are specialized to specific conditions, or because they are relicts [31]. Consistently, studies examining the predictive performances of SDMs and MSDMs for rare species suggest that gains in performance are context dependent [32]. 

interactions?

Inferring biotic interactions from co-occurrence patterns is a particularly hot topic in current ecological research [33-35]. In this context, some seminal articles have emphasized the potential of JSDMs to capture the signal of biotic interactions in the residual correlation matrix [36]. Although other authors entirely rejected this proposition [37], many are still left with the idea that the residual correlation matrix may 'hint at a biological interaction between species' [24] or 'inform about biotic constraints' [28]. Ongoing discussions turn around the scale mismatch between the true interactions and the modelled environment [37], the influence of missing predictors [38] and the symmetric constraint of correlation matrices [39] as important limitations of JSDMs, while others object that the signal that biotic interactions leave on co-occurrence data prevents any inference, whatever the method used [40,41]. Here, our argument focuses on a more fundamental limitation of JSDMs. Indeed, if the regression coefficients only estimate species' realized niches (question 1), not much of the signal of biotic interactions can remain in the residuals (even without any of the abovementioned problems) and what remains strongly depends on the characteristics of these interactions.

When considering two species $A$ and $B$ with overlapping fundamental niches (Figure 1.a) and assuming that $A$ is the strongest competitor, then B will be excluded from the overlapping area (Figure 1.b). The famous Barnacles in the low tide area are a typical example where Balanus (species A) excludes Chtamalus (species B) from large parts of its fundamental niche [42]. Applied to this data, SDMs, MSDMs and JSDMs will (wrongly) attribute the absence of species $B$ to the abiotic conditions. Since the realized niches entirely explain the negative correlation between the two species, no information on biotic interactions is left in the residuals, preventing JSDMs (and SDMs and MSDMs when correlating their residuals) to suggest a competitive interaction from the residual correlation matrix (the same logic applies for facilitation).

In contrast, let's assume that species A and B compete symmetrically, excluding each other about half the time in the overlapping region. An example is the unshaded reaches of Augusta Creek, Michigan (USA) (see [43], for a terrestrial example), where, at high velocity sites, the likelihood that a site will be dominated by the macroalga Cladophora glomerata or by epilithic microalgal lawn inhabited by several species of sessile grazers (e.g. the caddisflies Leucotrichia pictipes) is determined by who establishes first [44]. In this case, biotic interactions do not only affect the realized niches (that is decreased in magnitude, Figure 1.c), but also the species covariation around the expected distributions. So, the realized niches cannot fully explain the negative correlation between the species, and this part will appear in the residuals. Under the assumption of a well-specified model, JSDMs will identify the negative residual correlation between the species, which can truly be attributed to the competitive interaction between $A$ and $B$. Finally, notice that a common response to an unmeasured environmental covariate (e.g. both species prefer a warm climate) might lead to a positive correlation even if the two species do not interact $[38,45]$.

While abundance data may provide more informative than presence-absence to detect variations around the realized niches, environmental and biotic effects will still be confounded in the estimation of species responses to the 
environment (i.e. in $\boldsymbol{\beta}$ ). Therefore, when partitioning species covariance into shared environmental preferences and

262 residual co-occurrence patterns [24], one has to remember that the former are due to the realized niche and not due to 263 the fundamental one, with the consequences that the latter only reflects a small part of the signal of biotic interactions. 264 In conclusion, even if biotic interactions are an important process, their signal on co-distributions will be either fully or 265 partly hidden in $\boldsymbol{\beta}$.

As a statistical side note, we need to keep in mind that, even in the specific case that the residual correlation matrix $\boldsymbol{R}$ really captures an imprint of species interactions (which is unlikely for real data [40]), it represents the marginal correlations among the residuals and thus mixes the direct (e.g. competition) and indirect (e.g. a shared predator) associations between species. To conclude on direct associations between two species, we need to calculate the precision

271 matrix instead $\Omega=\boldsymbol{R}^{\mathbf{1}}$, that represents the (residual) partial correlation between species while controlling for the 272 effects of the other species $[46,47]$. 


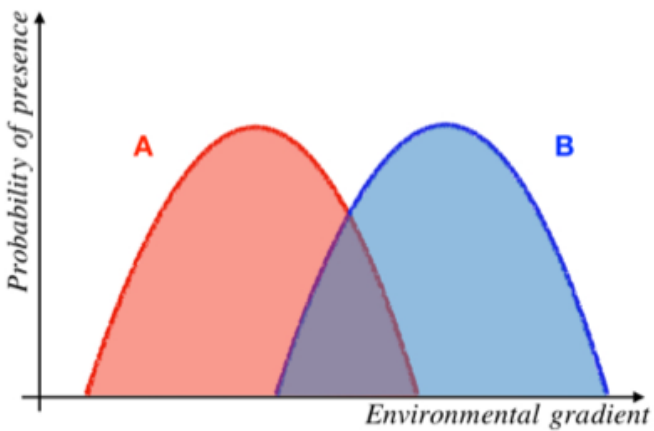

b)

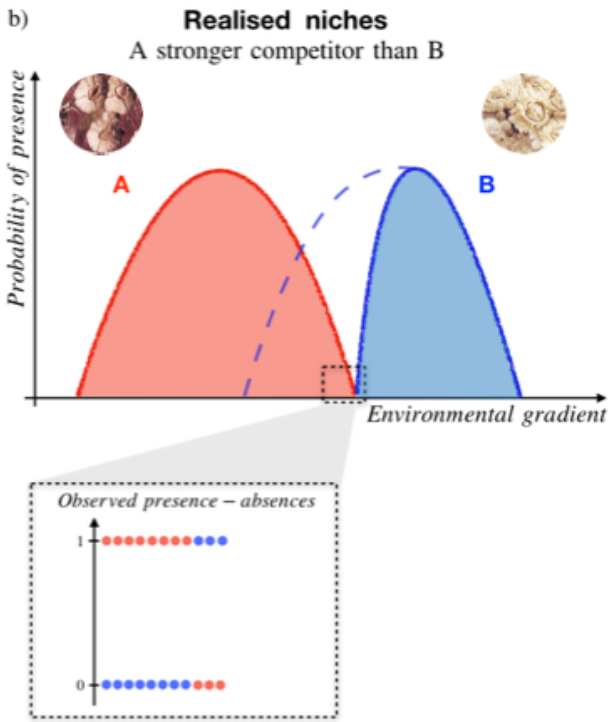

c)

A and B symmetric competitors

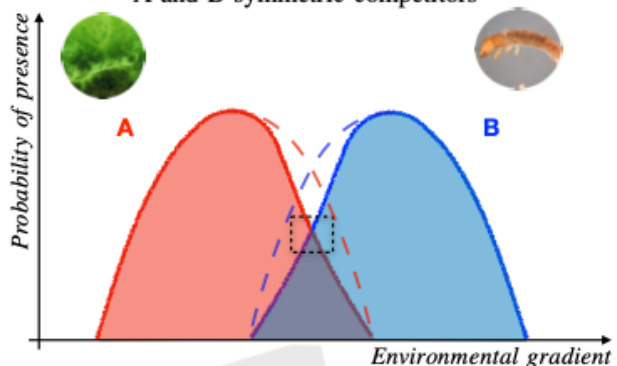

Environmental gradient

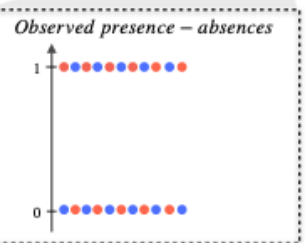

Figure 1. Effects of biotic interactions on species' niches. The top panel (a) shows the fundamental niche of two species ( $A$ and $B$ ). The bottom panels show two extreme scenarios of competition and the resulting realized niches (the fundamental niches are represented with the dashed line). SDMs, MSDMs and JSDMs retrieve the realized niches only. On the left (b), wherever the fundamental niches of $A$ and $B$ overlap, $A$ excludes $B$, even under weak abiotic conditions but still suitable for both species (e.g. Balanus and Chtamalus in [32]). The observed presences and absences in the interaction zone (the dashed rectangle) reflect this dichotomy due to competition exclusion, with little or no variation around the expected distribution where $A$ is present, and $B$ is absent. Since the realized niches entirely explain the negative correlation between $A$ and $B$, JSDMs will not identify a negative residual correlation. On the right (c), species $A$ and $B$ compete in a symmetric way, by excluding each other about half of the times where their niches overlap (Cladophora glomerata and Psychomyia flavida in [34]). If the expected distribution is the same for both species (their observed probability of occurrence in the conflict region is 0.5 ), their covariation around it is highly significant in terms of interactions, since the two species never co-occur. Here, JSDMs (but also MSDMs and SDMs when correlating their residuals) will detect a negative residual correlation since the realized niches do not fully explain the negative correlation between species.

\section{Box 3: An empirical example}

To elucidate the differences between SDMs and JSDMs in an empirical case-study, we focus on the response of alpine plants to snowmelt dates in Aravo (French Alps , [58]), as also done by [25]. We considered 65 species (all with more than 4 occurrences) at 75 sites, with snowmelt dates as the environmental covariate (linear and quadratic term, using orthogonal polynomials to reduce correlation among the covariates). The data are available from the $\mathrm{R}$ package ade4 [59]. To strictly focus on the effect of the residual correlation matrix on the estimates of the model, we avoid the confounding effects that can affect our results (e.g. choice of priors, different inference strategy, different implementation) by using the R package BayesComm [60], that allows us to choose whether residuals are considered as independent (multiple SDMs) or not (JSDM) and does not model the regression coefficients hierarchically (see Appendix 283 C for the code and further details). 
SDMs and JSDM estimated the same environmental niches. We can see almost no difference between the regression coefficients (Figure Ila, $R^{2}=0.987$ between the posterior means of the two models), and, in this case, the credible intervals are also very similar (see Appendix D). As a natural consequence, the marginal predictions are extremely close too $\left(R^{2}=0.996\right.$ between the posterior predictive means of the two models Figure $\left.\mathrm{llb}\right)$.

\section{a)}

$$
R^{2}=0.987
$$

Posterior mean of

the regression coefficients

总。

\section{Residual correlation matrix}

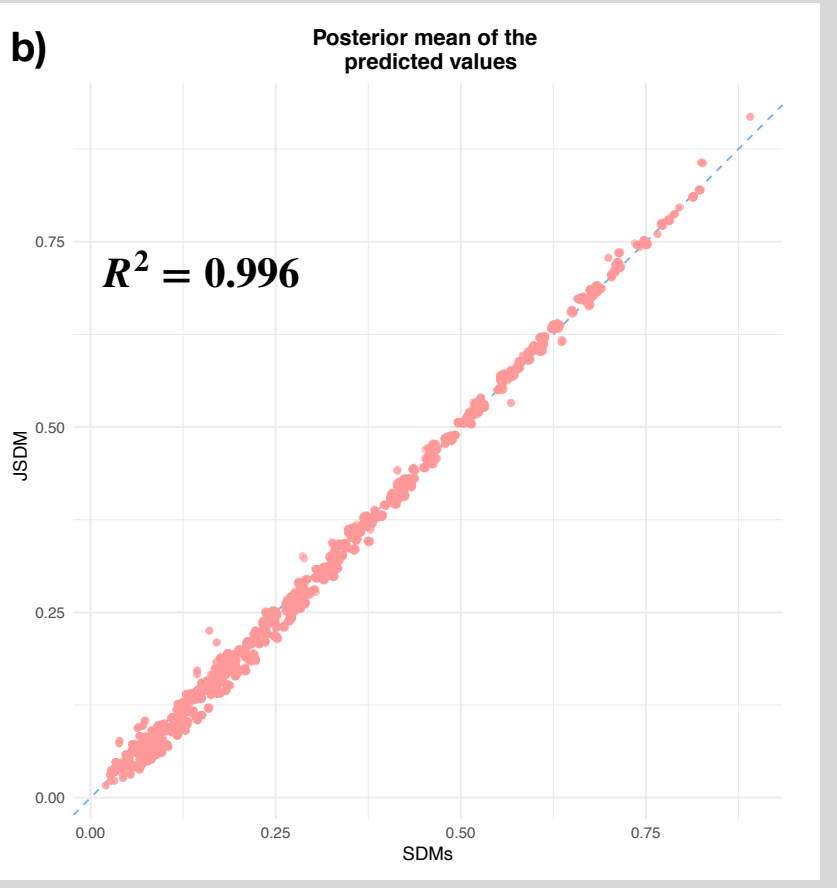

Figure I. On the left (a), relationship between the posterior means of the regression coefficients for all species estimated by SDMs on the $x$-axis and JSDM on the $y$-axis. Each point corresponds to a single coefficient (i.e. intercept, linear and quadratic term for snowmelt date) for a single modelled species. On the right (b), relationship between the posterior means of the predicted probability of presence. Each point corresponds to the predicted probability of presence of a single species at a single site. The blue dashed lines correspond to the 1:1 line.

We compared the correlation between the residuals of SDMs and the residual correlation matrix inferred by JSDM. Since a JSDM is a probabilistic model that allows error propagation, it is clearly preferable over multiple SDMs to infer a correlation matrix from the residuals. Here, we carry out this computation only to show the similarity between the two approaches. The residuals of the SDMs are calculated as the difference between the latent variables and the regression term, to stick to JSDMs definition of residuals (see Appendix D for other kinds of residuals). The residual correlation matrices estimated by SDMs and JSDM are very similar $\left(R^{2}=0.862\right.$ between the estimates of the two models, $95 \%$ credible intervals match in $98 \%$ of the cases, Figure II). 


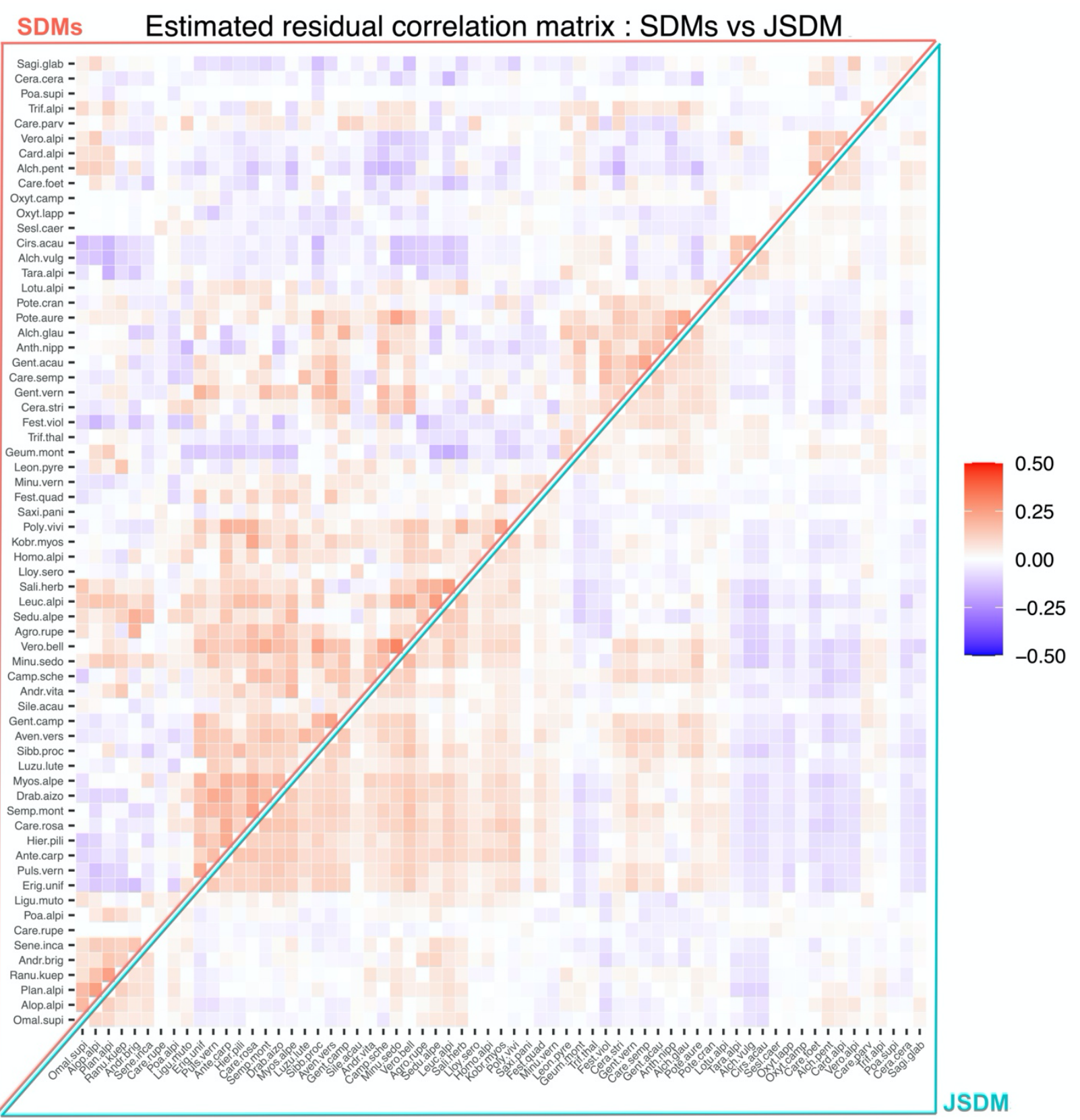

Figure I. Comparison of residual correlation matrices from multiple independent SDMs (post-hoc calculated) and a JSDM. SDMs residual correlations are represented in the upper triangular matrix, JSDM correlations in the lower triangular matrix. $R^{2}=0.862$ between the estimates of the two models, $95 \%$ credible intervals match in $98 \%$ of the cases (either both positive, or both negative, or both overlapping zero).

\section{Question 3: When and why do JSDMs outperform SDMs?}

310 One of the major objectives of species distribution models is to predict community compositions under new, eventually

311 future, abiotic conditions. For SDMs, MSDMs and JSDMs, the marginal prediction of each species (i.e. unconditionally

312 on the others) is only driven by whether the new environmental conditions are suitable for the species, even if the

313 marginal predictions of MSDMs (and JSDMs with hierarchical coefficients) can differ, for the reasons highlighted in

314 question 1. However, and importantly, this implies that all methods will project future species distributions without 
accounting for biotic interactions, although they are likely to play a critical role in the reorganization of communities as a result of global changes [4]. Since the estimated regression coefficients do not change whether species are modelled jointly or not, the marginal predictions do not change either, but have different uncertainties. In other words, fitting and predicting each species independently (SDMs), or with a JSDM, will lead to the same marginal predictions (as shown in Box 2, and see also Figure 2 of [48]). This explains why [29], [49] and [50] did not find clear differences in the predictive performance between JSDMs and SDMs neither at the species nor at the community level.

As a consequence, species richness predictions, that sum the mean marginal probabilities of SDMs vs. JSDMs, will inevitably coincide [51]. However, since the variance of a sum of correlated variables is not merely the sum of the variances, the residual correlation matrix does affect the uncertainty around the predicted richness. This is highlighted in the third box of [25], where the authors show that if the residual correlation across species was ignored (SDMs), the credible intervals were too narrow to capture the observed value of species richness.

The inferred residual correlation matrix still provides information on co-occurrence patterns that can be used to improve predictions. Indeed, JSDMs can leverage on the shared residual structure (that does not need be related to biotic interactions) to better estimate the probability of species co-occurrences and to provide joint and conditional predictions [52]. In other words, we should not interpret the residual correlation matrix, but rather exploit it.

When we commonly observe two co-occurring species, our expectation to see one when we see the other increases. This is what is called conditional prediction: the probability of presence of one (or more) species, given the presence, or absence, of one (or more) other species. JSDMs can exploit the residual correlation matrix to provide such predictions, where the observed species are basically used as predictor of the unobserved species. Conditional predictions can be of a great asset in several ecological applications. For instance, in invasion ecology, we could use JSDMs to determine the probability of invasive species to be present given the distribution of native species. Not only can they improve predictions, but they can also provide a better understanding of the studied system [49]. Studying how co-occurrence probabilities vary along environmental gradients, can also provide important knowledge on communities. Under the independence assumption of SDMs, the probability of co-occurrence is simply the product of marginal occurrence probabilities, but this estimate fails to integrate interspecific correlations. JSDMs are instead a potentially suitable tool for this task, since the probability of co-occurrence also depends on the residual correlations: positively correlated residuals lead to higher probability of co-occurrence than SDMs and vice-versa. Importantly, accounting for residual correlations to predict species co-occurrences inherently requires to have meaningful residuals that reflect underlying mechanisms (e.g. dispersal limitations, biotic interactions). In the extreme case of residual correlations completed driven by model error and/or misspecification, joint and conditional predictions might not improve, or even worsen, cooccurrence probabilities, especially when extrapolating in space and time.

\section{Concluding remarks}

The recent emergence of MSDMs and JSDMs has raised expectations to integrate some fundamental ecological processes in species distribution modelling, in particular to disentangle biotic interaction effects from environmental effects on 
species co-distributions. However, we show that these models do not account for biotic interactions when predicting distribution patterns, instead they infer correlations among taxa after accounting for environmental covariates. Therefore, they can only infer species' realized niches, and marginal predictions are not improved. We emphasize that we should not interpret the residual correlation matrix from a pure interaction perspective (whose ability to infer biotic interactions is strongly context dependent), but leverage on it, using conditional predictions, the under-exploited advantage of JSDMs. Hierarchical models, like MSDMs (or JSDMs with hierarchical effects) allow to test for the importance of traits and/or phylogeny and might bring interesting information notably for species that are difficult to detect, but the assumption behind these hierarchical effects need to be clearly understood by users.

\section{Outstanding Questions}

To what extent do biotic interactions leave an imprint in co-occurrence patterns to enable them to be distinguished from environmental effects? Under what conditions or types of interactions are these imprints detectable and what prior information would be needed to help the inference?

How can we better harness temporal data from multiple sources to exploit theory-based temporally-dynamic joint species distributions? Can dynamic JSDMs model species rich communities or would they be restricted to specific cases?

How can conditional dependencies in JSDMs or related graphical models be better used to provide conditional predictions for invasion risk assessment, re-introduction analyses or rare species modelling?

How can we account for biotic interactions when predicting species distribution and community compositions? How can we make best use of prior information on forbidden or known interactions?

\section{Acknowledgments}

We thank Frederic Gosselin, Frederic Mortier, Laura Pollock, Bjorn Reineking, and Stephane Robin for the insightful discus- sions on the properties of JSDMs. G.P., D.B., W.T., and T.M. were supported by the GAMBAS project funded by the Agence Nationale pour la Recherche (ANR-18-CE02-0025). W.T. and J.S.C. also acknowledge support from the Programme d'Investissement d'Avenir under project FORBIC (18-MPGA-0004). This work also received funding from the ERA-Net BiodivERsA - Belmont Forum, with the national funder Agence Nationale pour la Recherche (FutureWeb: ANR18-EBI4-0009) to W.T. and the National Science Foundation (NSF grant: 1854976) to J.S.C.

\section{References}

1 Pulliam, H.R. (2000) On the relationship between niche and distribution. Ecol. Lett. 3, 349-361

2

3

6

\author{
Lortie, C.J. et al. (2004) Rethinking plant community theory. Oikos 107, 433-438
}

Soberón, J. (2007) Grinnellian and Eltonian niches and geographic distributions of species. Ecol. Lett. 10, 11151123

Tylianakis, J.M. et al. (2008) Global change and species interactions in terrestrial ecosystems. Ecol. Lett. 11, 13511363

Thuiller, W. et al. (2013) A road map for integrating eco-evolutionary processes into biodiversity models. Ecol. Lett. 16, 94-105 
3887 Guisan, A. and Thuiller, W. (2005) Predicting species distribution: offering more than simple habitat models. Ecol. Lett. 8, 993-1009

Guisan, A. et al. (2017) Habitat Suitability and Distribution Models: With Applications in R, Cambridge University Press.

McCullagh, P. and Nelder, J.A. (1989) Generalized Linear Models, Second Edition, Chapman \& Hall.

Yates, K.L. et al. (2018) Outstanding challenges in the transferability of ecological models. Trends Ecol. Evol. 33, 790-802

Araújo, M.B. and Guisan, A. (2006) Five (or so) challenges for species distribution modelling. J. Biogeogr. 33, 16771688

Wisz, M. et al. (2013) The role of biotic interactions in shaping distributions and realised assemblages of species: Implications for species distribution modelling. Biol. Rev. Camb. Philos. Soc. 88, 15-30

Guisan, A. and Rahbek, C. (2011) SESAM - a new framework integrating macroecological and species distribution models for predicting spatio-temporal patterns of species assemblages. J. Biogeogr. 38, 1433-1444

Calabrese, J.M. et al. (2014) Stacking species distribution models and adjusting bias by linking them to macroecological models. Glob. Ecol. Biogeogr. 23, 99-112

Staniczenko, P.P.A. et al. (2017) Linking macroecology and community ecology: refining predictions of species distributions using biotic interaction networks. Ecol. Lett. 20, 693-707

D’Amen, M. et al. (2018) Improving spatial predictions of taxonomic, functional and phylogenetic diversity. J. Ecol. $106,76-86$

Ovaskainen, O. and Soininen, J. (2011) Making more out of sparse data: hierarchical modeling of species communities. Ecology 92, 289-295

Pollock, L.J. et al. (2012) The role of functional traits in species distributions revealed through a hierarchical model. Ecography (Cop.). 35, 716-725

Jamil, T. et al. (2013) Selecting traits that explain species-environment relationships: a generalized linear mixed model approach. J. Veg. Sci. 24, 988-1000

Brown, A.M. et al. (2014) The fourth-corner solution - using predictive models to understand how species traits interact with the environment. Methods Ecol. Evol. 5, 344-352

Carboni, M. et al. (2018) Functional traits modulate the response of alien plants along abiotic and biotic gradients. Glob. Ecol. Biogeogr. 27, 1173-1185

Harris, D.J. (2015) Generating realistic assemblages with a joint species distribution model. Methods Ecol. Evol. 6, 465-473

Vanhatalo, J. et al. (2020) Additive multivariate Gaussian processes for joint species distribution modeling with heterogeneous data. Bayesian Anal. 15, 415-447

Pollock, L.J. et al. (2014) Understanding co-occurrence by modelling species simultaneously with a Joint Species Distribution Model (JSDM). Methods Ecol. Evol. 5, 397-406

Warton, D.I. et al. (2015) So Many Variables: Joint Modeling in Community Ecology. Trends Ecol. Evol. 30, 766779

Ovaskainen, O. et al. (2017) How to make more out of community data? A conceptual framework and its implementation as models and software. Ecol. Lett. 20, 561-576

Clark, J.S. et al. (2017) Generalized joint attribute modeling for biodiversity analysis: median-zero, multivariate, multifarious data. Ecol. Monogr. 87, 34-56 
28 Wilkinson, D.P. et al. (2019) A comparison of joint species distribution models for presence--absence data. Methods Ecol. Evol. 10, 198-211

29 Norberg, A. et al. (2019) A comprehensive evaluation of predictive performance of 33 species distribution models at species and community levels. Ecol. Monogr. 89, 834- 848

30 Tikhonov, G. (2018) , Bayesian latent factor approaches for modeling ecological species communities. , Helsinki : Helsingin yliopisto,

31 Gaston, K.J. (1994) What is rarity? In Rarity pp. 1-21, Springer

32 Nieto-Lugilde, D. et al. (2018) Multiresponse algorithms for community-level modelling: Review of theory, applications, and comparison to species distribution models. Methods Ecol. Evol. 9, 834-848 Morales-Castilla, I. et al. (2015) Inferring biotic interactions from proxies. Trends Ecol. Evol. 30, 347-356 Sander, E. et al. (2017) Ecological Network Inference From Long-Term Presence-Absence Data. Sci. Rep. 7, 7154 Freilich, M. et al. (2018) Species co-occurrence networks: Can they reveal trophic and non-trophic interactions in ecological communities? Ecology 99, 690-699

Ovaskainen, O. et al. (2016) Using latent variable models to identify large networks of species-to-species associations at different spatial scales. Methods Ecol. Evol. 7, 549-555

Clark, J.S. et al. (2014) More than the sum of the parts: forest climate response from joint species distribution models. Ecol. Appl. 24, 990-999

Kissling, W.D. et al. (2012) Towards novel approaches to modelling biotic interactions in multispecies assemblages at large spatial extents. J. Biogeogr. 39, 2163-2178

Dormann, C.F. et al. (2018) Biotic interactions in species distribution modelling: 10 questions to guide interpretation and avoid false conclusions. Glob. Ecol. Biogeogr. 27, 1004-1016

Blanchet, F.G. et al. (2020) Co-occurrence is not evidence of ecological interactions. Ecol. Lett. 23, 1050-1063

Holt, R.D. (2020) Some thoughts about the challenge of inferring ecological interactions from spatial data. Biodivers. Informatics 17, 61-85

Connell, J.H. (1961) The Influence of Interspecific Competition and Other Factors on the Distribution of the Barnacle Chthamalus Stellatus. Ecology 42, 710-723

Palmer, T.M. et al. (2003) Competition and Coexistence: Exploring Mechanisms That Restrict and Maintain Diversity within Mutualist Guilds. Am. Nat. 162, S63-S79

Hart, D. (1992) Community organization in streams: the importance of species interactions, physical factors, and chance. Oecologia 91, 220-228

Momal, R. et al. (2020) Accounting for missing actors in interaction network inference from abundance data. arXiv DOI: stat.AP/2007.14299

Harris, D.J. (2015) Generating realistic assemblages with a joint species distribution model. Methods Ecol. Evol. 6, 465-473

Popovic, G. et al. (2019) Untangling direct species associations from indirect mediator species effects with graphical models. Methods Ecol. Evol. 10, 1571-1583

Chen, D. et al. (2017), Deep multi-species embedding. , in IJCAI International Joint Conference on Artificial Intelligence, pp. 3639-3646

Zurell, D. et al. (2020) Testing species assemblage predictions from stacked and joint species distribution models. J. Biogeogr. 47, 101-113 
$46950 \quad$ Caradima, B. et al. (2019) From individual to joint species distribution models: A comparison of model complexity 470 and predictive performance. J. Biogeogr. 46, 2260-2274

47151 Gelfand, A.E. and Shirota, S. (2019) Clarifying species dependence under joint species distribution modeling. 472 bioRxiv DOI: 10.1101/744359

47352 Wilkinson, D.P. et al. (2020) Defining and evaluating predictions of joint species distribution models. Methods $474 \quad$ Ecol. Evol. https://doi.org/10.1111/2041-210X.13518

Barve, N. et al. (2011) The crucial role of the accessible area in ecological niche modeling and species distribution modeling. Ecol. Modell. 222, 1810-1819 Hutchinson (1957) Population studies: Animal ecology and demography. Bull. Math. Biol. 53, 193-213 Ricklefs, R.E. (2010) Life-history connections to rates of aging in terrestrial vertebrates. Proc. Natl. Acad. Sci. $107,10314-10319$

Albert, J.H. and Chib, S. (1993) Bayesian analysis of binary and polychotomous response data. J. Am. Stat. Assoc. 88, 669-679

Choler, P. (2005) Consistent shifts in alpine plant traits along a mesotopographical gradient. Arct. Antarct. Alp. Res. 37: 444-453 Softw. 22:1-20. 